\title{
Estratégias de Prevenção do Suicídio em Médicos: Revisão Sistemática de Literatura
}

\author{
Ivete Hiroko Kawasaki* \\ Pedro Eduardo Silva Ambra**
}

\begin{abstract}
Resumo
O objetivo deste estudo foi realizar uma revisão sistemática da literatura para identificar e analisar as estratégias de prevenção do suicídio utilizadas em médicos. A classe médica é uma das que mais cometem suicídio, é um problema mundial e de saúde pública. A Organização Mundial da Saúde - OMS e outras organizações internacionais têm trabalhado juntas para reduzir o suicídio, no entanto, esse fenômeno não foi reduzido entre os médicos. A revisão sistemática foi realizada em cinco base de dados (PubMed, LILACS, SciELO, PsycINFO, Cochrane) com estudos publicados referentes à prevenção do suicídio em médicos nos últimos 11 anos (2008-2018). Foram encontrados apenas 05 artigos que atenderam os critérios de inclusão. Observou-se que não há homogeneidade ou sistematização clara nas intervenções, houve pouca participação dos médicos nos programas de prevenção do suicídio devido ao estigma do transtorno mental e ao suicídio, mas que foram eficazes para aqueles que procuraram por ajuda.
\end{abstract}

Palavras-chave: médico; suicídio; prevenção do suicídio.

\section{Suicide Prevention Strategies in Physicians: Systematic Literature Review}

\begin{abstract}
The objective this study was to conduct a systematic literature review to identify and analyze suicide prevention strategies used by physicians. The medical profession is one of the most committing suicide; it is a worldwide and public health problem. WHO (World Health Organization) and other international organizations have worked together to reduce suicide worldwide, but this phenomenon has not been reduced among doctors. The review was performed in five electronic databases (PubMed, LILACS, SciELO, PsycINFO, Cochrane) to systematically find and present published studies on suicide prevention in physicians in the last 11 years (2008-2018). Despite the relevance of the question, only 05 articles that met the inclusion criteria were found. It was observed that there is no clear homogeneity or systematization of interventions, there was little participation of doctors in suicide prevention programs due to the stigma of mental disorder and suicide, but they were effective for those seeking help.

Keywords: Physician, Suicide, Suicide prevention.
\end{abstract}

* Médica pela UNESP/Botucatu, Mestre em Psicossomática pela Universidade Ibirapuera e Médica anestesiologista na UNIFESP/SP.

** Professor do Curso de Psicologia da Pontifícia Universidade Católica de São Paulo (PUC-SP), Docente Colaborador e Orientador do Programa de Pós-graduação do Departamento de Psicologia Social da Universidade de São Paulo (USP). Graduação em Psicologia pela USP, mestrado e doutorado em Psicologia Social pela mesma instituição (Bolsa CNPq) e doutorado em dupla-titulação em Psychanalyse et Psychopathologie pela Université de Paris (Bolsa CAPES-PDSE). Orientador da Dissertação apresentada à Universidade Ibirapuera - UNIB, para obtenção do Título de Mestre em Psicologia, área de concentração em Psicossomática. 


\section{Introdução}

O suicídio, ato intencional e voluntário de tirar a própria vida (Brasil, 2009), segundo Cassorla (1984) é a morte de si mesmo, ato intencional de matar a si mesmo (Botega, 2015). Há décadas tem sido motivo de preocupação da Organização Mundial da Saúde - OMS, (2014), do Ministério da Saúde (MS, 2014), pois é um grande problema de saúde pública. Sua prevenção é uma prioridade para a OMS (2014). Scavacini (2018) propõe estimular a comunicação sobre o suicídio, desenvolver mensagens dirigidas a públicos específicos, promover campanhas com famosos e influenciadores para atingir mais pessoas e sensibilização com a problemática para que não ocorra divulgação de suicídios espetaculares e a ocorrência de outras mortes por imitação ou contágio. Imitação é o processo pelo qual um suicida exerce um efeito modelador em suicídios subsequentes. Contágio é o processo pelo qual um determinado suicídio facilita a ocorrência de outros suicídios, a despeito do conhecimento direto ou indireto do suicídio prévio (OMS, 2000).

A cada 40 segundos ocorre um suicídio no mundo. A cada ano, cerca de 800 mil pessoas tiram a própria vida (OMS, 2014; OMS, 2018) e o número de tentativas é de 10 a 20 vezes maiores.

Há indicativos de o suicídio ter elevada relação com quadros psiquiátricos (Center, Davis, Detre, Ford, Hansbrough, Hendin, Laszlo, Litts, Mann, Mansky, Michels, Miles, Proujansky, Reynolds \& Silverman, 2003; Chachamovich, Stefanello, Botega \& Turecki, 2009; Gerada, 2018; Hawton, Malmberg \& Simkin, 2004) na qual a depressão maior se destaca. Depressão passou a ser utilizado durante o século XIX. Classificado de acordo com o CID-10 como transtorno de humor. A depressão pode ser leve, moderado e grave (maior) de acordo com o número de episódios depressivos, intensidade e importância clínica. Os sintomas da depressão são: rebaixamento do humor, perda do interesse e da capacidade de experimentar o prazer; redução da energia e diminuição da atividade; diminuição da capacidade de concentração e fadiga acentuada; diminuição da autoestima e autoconfiança e frequentes ideias de culpa; sintomas somáticos como a lentidão psicomotora acentuada ou agitação; perda de apetite, perda de peso e perda da libido. Depressão leve: 2 a 3 sintomas estão presentes, o indivíduo é capaz de exercer a maior parte das atividades. Depressão moderada: presença de 4 ou mais sintomas, apresenta dificuldades para atividades de rotina. Depressão grave ou maior com sintomas somáticos: vários dos sintomas estão presentes, são angustiantes e acentuados, ideação suicida e atos suicidas são comuns e sintomas somáticos. Depressão grave ou maior com sintomas psicóticos: depressão acompanhada de alucinações, delírios, lentidão psicomotora, estupor (desapare- cimento da sensibilidade ao meio ambiente e da faculdade de exibir reações motoras), impossibilidade de relações sociais, desidratação e/ou desnutrição e risco de suicídio.

Os transtornos mentais são tratáveis desde que sejam reconhecidos (OMS, 2000b; Botega, Werlang \& Macedo, 2006). Para uma efetiva prevenção, é necessária uma ampla estratégia com a participação de vários setores da saúde.

Um fenômeno que tem se observado é o aumento do número de suicídio nas variadas profissões, e os médicos, profissionais de saúde e estudantes de medicina receberam destaque (Meleiro, 1998; Millan \& Arruda, 2008), principalmente nos Estados Unidos, país que apresenta em suas estatísticas elevada taxa de suicídio. Cerca de 300-400 médicos morrem por suicídio a cada ano (AFSP, 2015; Gold, Sen \& Schwenk, 2013; Sen, Kranzler, Krystal, Speller \& Chan, 2010), no qual 1 médico morre por suicídio a cada dia. Nos artigos científicos relacionados a suicídio em médicos, como profissão isolada, as referências mostram que a taxa é maior do que em outras profissões (American Foundation for Suicide Prevention (AFSP), 2015, 2018; Gunter, 2016; Hawton, Malmberg \& Simkin, 2004; Sheikhmoonesi \& Zarghami, 2014). A maioria dos autores baseia-se no artigo publicado pela revista JAMA em junho de 2003, Confronting Depression and Suicide in Physicians, a Consensus Statement (Center, et al., 2003). A publicação apresentou as possíveis causas do suicídio de médicos, discussões sobre o tratamento e conhecimento das barreiras enfrentadas por profissionais que dificultam a procura por ajuda. O estudo de Center et al. (2003) revelou que mais de $90 \%$ das pessoas que morreram por suicídio, tinham algum transtorno mental no qual a depressão maior ou transtorno bipolar receberam destaque. Transtorno Bipolar Consiste tipicamente em episódios de mania e depressão, separados por períodos de humor normal. Os episódios de mania envolvem humor elevado ou irritado, excesso de atividade, pressão de fala, autoestima inflada e uma menor necessidade de sono. As pessoas que têm episódios de mania, mas não experimentam episódios depressivos, também são classificadas como tendo transtorno bipolar. Fonte: https:// www.paho.org $/ \mathrm{bra} /$ index.php?option $=\mathrm{com}_{-}$content\& view $=$ article $\& i d=5652$ :folha-informativa-transtornos-mentais\&Itemid $=839$

O suicídio é prevenível e tratável (Meleiro, 1998; Santa \& Cantilino, 2016), no entanto, há pouco conhecimento sobre sua prevenção, tratamento e eficácia para a classe médica.

A prevalência na literatura médica de suicídio entre os médicos, assim como a depressão, uso de substâncias psicoativas (droga e álcool), estresse e burnout é alta. É um reflexo do seu melhor conhecimento e habilidade 
técnica na decisão de como consumar a morte.

Na medicina, há uma história de 150 anos de suicídio em médicos na América (Legha, 2012) e no mundo divulgadas no decorrer dos anos em vários meios de comunicações disponíveis. O seu tratamento é viável e eficaz, mas a luta tem sido perdida devido principalmente ao estigma da doença mental e do suicídio.

Este estudo tem a proposta de identificar e avaliar quais estratégias de prevenção e tratamento de suicídio em médicos são utilizados para este grupo de profissionais e se são eficazes, por meio de uma revisão sistemática de artigos publicados sobre o assunto.

\section{Método}

A pesquisa foi estruturada conforme a estratégia metodológica Prefered Reporting Itens for Systematic Reviews and Meta-Analyses - PRISMA (Moeher \& Altman, 2010). Realizou-se um levantamento de artigos nas bases de dados LILACS, SciELO Brasil, PubMed, Cochrane Library e PsycINFO, nas quais foram realizadas diferentes estratégias de buscas utilizando os descritores e combinações booleanas (AND, OR) com o seguinte conjunto de palavras-chave: médico ou residente ou profissional de saúde, suicídio ou tentativa de suicídio, prevenção ou prevenção do suicídio. As palavras chaves residente e profissional de saúde foram incluídas para ampliar a pesquisa no campo da designação profissional como médico. Para esta revisão sistemática foram incluídos artigos publicados no período de 2008 a 2018, foram selecionados inicialmente artigos com texto completo e resumo, publicações em inglês, português e espanhol. O passo seguinte foi a exclusão de artigos por multiplicidade. No idioma em inglês, a pesquisa ocorreu nas duas formas gramaticais para prevenção do suicídio, "suicide prevention" e "prevention of suicide".

Os critérios de exclusão foram: a) dissertações de mestrados e doutorados; b) artigos que analisam somente estudantes de medicina; c) artigos de prevenção do suicídio em médicos aposentados.

Foram encontradas 1545 referências, seguiu-se uma seleção de trabalhos científicos primeiramente por inclusão de artigos publicados no período de 01 de janeiro de 2008 a 31 de dezembro de 2018. Aplicando-se inicialmente esses critérios, foram excluídas 773 referências, restando, então, 772. Foram escolhidos somente textos completos e resumos que resultaram na exclusão de 193 artigos nos quais as dissertações estão incluídas. O próximo critério de exclusão foi o idioma, foram retirados 22 textos, resultando 557 artigos. O próximo passo foi a exclusão de 209 artigos repetidos nas bases pesquisadas restando 348 artigos para leitura crítica dos títulos e resumos. Em seguida foram excluídos 344 artigos que não preenchiam os critérios de elegibilidade. $\mathrm{Na}$ pesquisa realizada na base eletrônica da LILACS com a combinação em espanhol "médico AND suicídio AND prevención" foi encontrado duplicidade de um artigo sobre Suicídio/Suicide de Martin Nizama Valladolid (2011), publicado na mesma revista científica (online), com a mesma paginação. As eliminações resultaram em quatro artigos que preencheram os requisitos de inclusão. Após leitura de referências de artigo elegível, foi incluído mais um texto para análise. O processo de coleta está presente na Figura 1.
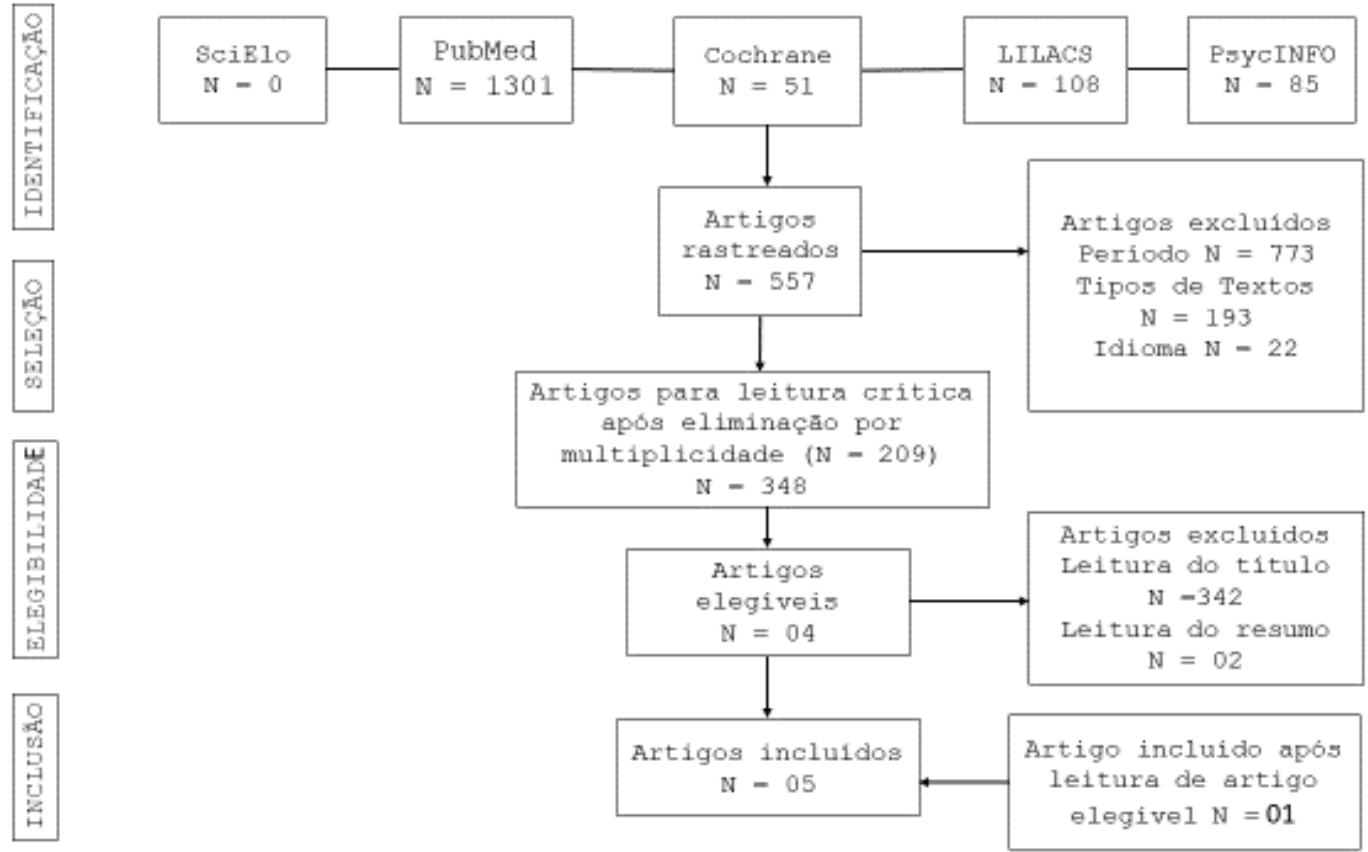

Figura 1 - Prisma da pesquisa 
Os artigos foram obtidos através das bases de dados e, quando não disponibilizados na íntegra em tais bases, foram acessados através do serviço de bibliotecas da Universidade de São Paulo - Instituto de Psicologia e através de sites que oferecem acesso gratuito a artigos científicos.

\section{Resultados}

A revisão sobre as estratégias de prevenção do suicídio em médicos resultou em cinco artigos com diferentes abordagens para atingir e obter maior participação dos médicos de várias formações e especialidades. Foram 04 artigos publicados nos Estados Unidos e 01 artigos no México. Na base de dados da SciELO Brasil não foram

Quadro 1 - Seleção dos artigos

\begin{tabular}{|l|l|l|}
\hline AUTOR/ANO & PAÍS & POPULAÇ̃̃O \\
\hline $\begin{array}{l}\text { Moutier, Norcross, Jong, Norman, Kirby, McGuire e } \\
\text { Zisook (2012) - 2009-2010 }\end{array}$ & USA & $\begin{array}{l}\text { Alunos med/farm n= 498/240 } \\
\text { Docentes } \mathrm{n}=1300 \\
\text { Med/Res/Fellows } \mathrm{n}=822\end{array}$ \\
\hline $\begin{array}{l}\text { Haskins, Carson, Chang, Kirshnit, Link, Navarra, Scher, } \\
\text { Sciolla, Uppington e Yellowlees (2016) - 2013-2014 }\end{array}$ & USA & $\begin{array}{l}\text { Res/Fellows } \mathrm{n}=946 \\
\text { Docentes } \mathrm{n}=918\end{array}$ \\
\hline $\begin{array}{l}\text { Guille, Zhao, Krystal, Nichols, Brady e Sen (2015) } \\
\text { 2009-2010 / 2011-2012 }\end{array}$ & USA & Residentes $\mathrm{n}=320$ \\
\hline Ey, Moffit, Kinzie e Brunett (2016) - 2011 & USA & $\begin{array}{l}\text { Res/Fellows } \mathrm{n}=906 / 116 \\
\text { Docentes } \mathrm{n}=1400 / 23\end{array}$ \\
\hline Jiménez-López e Arenas Osuna (2017) - 2011-2015 & México & Residentes $\mathrm{n}=594$ \\
\hline
\end{tabular}

encontrados artigos científicos brasileiros publicados no país sobre o tema (Quadro 1)

Os artigos apresentam transtornos mentais como um denominador comum e o estigma dos médicos em relação a esses transtornos e ao suicídio como barreiras para o tratamento e sua prevenção. Os médicos admitem preocupações relacionados a confidencialidade; privacidade; custos de tratamento; limitação de tempo; incerteza sobre a eficácia; notificações de tratamento em credenciamento, licenciamento médico e seguro de vida e profissional e; principalmente, apreensão quanto ao estigma do transtorno mental na profissão. As publicações estão voltadas para superar esses obstáculos e proporcionar terapia adequada para o médico.

Dois artigos, de Moutier, et al. (2012) e Haskins, et al. (2016) realizados na University of California, San Diego School of Medicine e na University of California, Davis Health System, Sacramento respectivamente, realizaram a mesma abordagem em diferentes períodos e obtiveram o mesmo resultado. No estudo realizado em 2012 foram inclú́dos os alunos de medicina e da farmacologia, médicos do corpo clínico, médicos residentes e docentes da instituição médica. Em 2016, Haskins et al. replicou o estudo de Moutier et al. (2012) mas excluiu os alunos. A abordagem foi realizada via convite eletrônico para participar do programa no website da instituição sobre bem-estar e prevenção do suicídio. Poucos aceitaram o convite, mas a maior parte dos participantes apresentou risco moderado a grave para depressão e/ou suicídio. No entanto, poucos aceitaram ser encaminhados para tratamento. Aqueles que concordaram com a terapia relataram melhora do estado mental.

No artigo publicado por Moutier et al. (2012) foi realizado convites eletrônicos para participação do estudo, foram incluídos além dos médicos residentes/ fellows (822) e docentes (1300), os alunos de medicina e farmacologia. Concordaram participar do estudo $8 \%$ de 822 e $11 \%$ de 1300 . Após o rastreamento, apenas $2 \%$ e $1 \%$, respectivamente, foram encaminhados para tratamento com especialistas com melhora do quadro. $\mathrm{Na}$ réplica realizada por Haskins et al. (2016) somente os residentes/fellows (946) e docentes (918) foram convidados para participar do programa. O aceite do convite foi de $10 \%$ e $5 \%$ respectivamente e apenas $1 \%$ de 918 e $0,7 \%$ aceitaram ser encaminhados para um especialista e relataram melhora dos sintomas de depressão e/ou suicídio. No rastreamento do programa, foram identificados participantes com transtorno mental 
e risco de suicídio importante, mas poucos prosseguiram com o tratamento.

O estudo realizado por Guille et al. (2015) foi direcionado para médicos residentes que estivessem iniciando a residência médica em 2 hospitais universitários, Yale University e University of Southern California, durante os anos de 2009-2010 e 2011-2012. Foi enviado um e-mail 3 meses antes do início da residência médica, convidando-os para participar do estudo, que avaliou a intervenção através da Terapia cognitivo-comportamental baseada na web ou internet (TCCi) para prevenir ideação suicida. Dos 320 convites eletrônicos, 199 médicos residentes/ estagiários aceitaram, que corresponde a participação de 62,2\% dos residentes. O item 9 do Patient Health Questionnaire-9 (PHQ-9) foi utilizado para avaliar a ideação suicida (pensamentos de que você estaria melhor morto ou se machucando de alguma forma) antes do início da residência médica e durante o ano. Os participantes foram divididos em 2 grupos: Grupo Controle de Atenção (GCA) e o grupo da TCCi. O GCA recebeu um e-mail uma vez por semana, durante quatro semanas com informações sobre transtornos mentais, incluindo sintomas de depressão, suicídio e referências para obter tratamento local de saúde mental. O grupo da TCCi foi encaminhado para um site da intervenção (http://moodgym.anu. edu.au $)^{1}$ obtido por um nome de usuário fornecido no e-mail. Depois do acesso, os participantes criaram uma senha exclusiva conhecida apenas por eles, mas permitindo aos criadores do site o rastreamento da conclusão do módulo. Esse estudo mostrou que os participantes da TCCi apresentaram 60\% menos chances de relatar ideação suicida durante um ano de estágio que o grupo de controle da atenção.

O trabalho publicado por Ey, Moffit, Kinzie e Brunett (2016) apresenta um programa da Oregon Health \& Science University, Graduate medical Education, o Resident and Faculty Wellness Program (RFWP). É um programa de bem-estar do residente e do corpo docente. Esse artigo avalia a satisfação, a viabilidade e utilização dos usuários relacionados ao programa de prevenção do bem-estar e suicídio para residentes, fellows e professores. Uma pesquisa com os participantes, 116 residentes/ fellows de 906 e 23 de 1400 docentes, aponta satisfação com o programa desenvolvido que inclui workshops de promoção de bem-estar; espaço físico com uma equipe de

MoodGYM é um programa de terapia cognitivo comportamental para prevenção e redução dos sintomas de ansiedade e depressão. No referido artigo foi utilizado para reduzir a ideação suicida. Cf. (http://moodgym. anu.edu.au) profissionais, psiquiatras e psicólogos, com experiência no tratamento de médicos e não estejam envolvidos na avaliação do desempenho dos residentes e docentes; triagem de prevenção de suicídio oferecidas inicialmente on-line e encaminhadas para tratamento individual se necessário; almoços mensais com grupos de residentes; abordagens individuais de coaching; aconselhamento psicológico, tais como terapia cognitivo-comportamental, meditação e tratamentos breves; avaliação psiquiátrica; controle medicamentoso; consultas com líderes do programa, médicos da educação e preceptor dos residentes sobre a angustia dos trainees/docentes encaminhamentos para serviços especializados (avaliações neuropsicológicas, internações e avaliação para exercer a função). A pesquisa mostra a satisfação de $97 \%$ dos residentes/fellows e de $88 \%$ dos docentes quanto ao programa da instituição. $\mathrm{O}$ artigo não fornece informações sobre taxas dos participantes com risco de suicídio e transtorno mental.

No artigo de Jiménez-López e Arenas Osuna (2017), os autores preocupados com casos de suicídio, 10 suicídios de 2000 a 2009, no Hospital de Especialidades “Dr. Antonio Fraga Mouret", Centro Médico Nacional La Raza (HECMR), criaram a primeira Clínica de Saúde Mental para Médicos Residentes (CSM-MR) direcionada para a prevenção do suicídio e depressão, tratamento de transtornos mentais e promoção da saúde mental. A CSM-MR tem o propósito de melhorar a saúde mental durante a residência, pretende identificar $90 \%$ dos residentes vulneráveis para depressão e suicídio no início do estágio, prevenir os suicídios, diminuir os erros médicos, proporcionar tratamento a $95 \%$ dos residentes que desenvolveram transtorno mental. A estratégia inicial para identificar os residentes foi aplicar questionários sobre depressão, risco de suicídio e uso de substâncias psicoativas no momento da matrícula para o estágio. A folha frontal dos questionários especificava a confidencialidade e o arquivamento dos dados na CSM-MR. Os médicos residentes eram encaminhados para a CSM conforme a pontuação das respostas dos residentes; indicação do chefe da disciplina e solicitação pelo próprio estagiário. Esse artigo mostrou o melhor resultado, no período do estudo que variou de 2011 a 2015 não ocorreram suicídios e os residentes com elevado risco para o suicídio receberam intervenção da equipe da CSM-MR.

\section{Discussão}

A revisão sobre as estratégias de prevenção do suicídio em médicos resultou em cinco artigos com diferentes abordagens para atingir e obter maior participação dos 
médicos de várias formações e especialidades. Diferentemente do que se poderia supor, a pequena quantidade de trabalhos encontrada na pesquisa causa surpresa, pois é um tema que tem causado preocupação há mais de 150 anos (Legha, 2012) na comunidade médica e na população geral.

Os artigos apresentaram estratégias isoladas para atender as necessidades das instituições que podem ser considerados como ensaios bem conduzidos de programas direcionados para os médicos, mas não são consideradas estratégias universais de prevenção do suicídio.

Todos os artigos relatam o transtorno mental, principalmente a depressão, como o maior responsável pelas altas taxas de suicídio e a identificação de barreiras que limitam o acesso ao tratamento psicológico ou psiquiátrico. Essas barreiras incluem a preocupação com a confidencialidade; privacidade; custos do tratamento; tempo limitado; incerteza sobre a eficácia; perguntas sobre a notificação de tratamento em credenciamento, licenciamento e seguro de vida e invalidez; e o estigma que os profissionais de saúde têm em relação aos transtornos mentais. A auto prescrição, recorrer à família em busca de ajuda ou "lutar em silêncio" São fatores que favorecem o médico a gerenciar os problemas por conta própria.

As propostas levantadas nos artigos visam romper essas barreiras, tratar os transtornos mentais e o suicídio.

Para romper a barreira da confidencialidade e privacidade, os estudos de Moutier et al. (2012), Haskins et al. (2016), Guille et al. (2015) e Ey, Moffit, Kinzie e Brunett (2016) criaram programas nos quais as informações dos médicos são criptografadas por um site online seguro da instituição, os participantes são identificados apenas por um número não codificável, os médicos foram convidados eletronicamente para participar dos programas apresentados. Moutier et al. (2012) e Haskins et al. (2016) desenvolveram o programa através do Projeto Médico de Prevenção da Depressão e do Suicídio desenvolvido pela American Foundation for Suicide Prevention (AFSP) em 2002. No artigo de Jiménez-López e Arenas Osuna (2017), não está claro se houve convite formal e consentimento dos residentes para participarem do programa proposto de tratamento e intervenção. Os estudos de Moutier et al. (2012), Haskins et al. e Ey, Moffit, Kinzie e Brunett (2016) solicitaram o consentimento dos participantes para os programas de bem-estar e prevenção do suicídio. Guille et al. (2012) não necessitaram do consentimento dos médicos residentes.

Moutier et al. (2012) e Haskins et al. (2016) optaram pela não intervenção mesmo com risco grave de depressão e/ou consumação do suicídio para não perder a credibilidade do programa em relação a confiabilidade e privacidade. No estudo realizado por Ey, Moffit, Kinzie e Brunett (2016), os autores divulgariam as informações nos casos de risco iminente de danos a si ou aos outros. No artigo publicado por Jiménez-López e Arenas Osuna (2017), os responsáveis pela CSM-MR intervinham na condução dos residentes com alto risco para o suicídio, no entanto o artigo não esclarece se os residentes foram informados da atuação ativa dos responsáveis pelo programa.

O estudo de Guille et al. (2015) demonstrou que um programa gratuito, fácil e acessível de TCCi diminui ideação suicida entre os médicos residentes. $\mathrm{O}$ uso de ferramentas baseadas na internet ou na Web traz benefícios sobre o tratamento em pessoas que necessitam de cuidado mental que incluem maior confidencialidade; baixo custo; fácil acessibilidade; flexibilidade no momento do acesso; e ferramentas de autogerenciamento. Em conjunto, o programa TCCi mostrou-se viável, eficaz e aceitável para a prevenção de ideação suicida entre os médicos residentes. É um programa que não necessita de um especialista para o tratamento, porém essa abordagem não é recomendada em pacientes com depressão grave e/ou risco elevado de suicídio. $\mathrm{O}$ artigo apresentou uma participação grande dos médicos residentes, cerca de $62,2 \%$, bem acima da média que geralmente é de 10-20\% nos recursos tradicionais de saúde mental. A maior participação pode ter ocorrido também pelo recebimento em certificados de presente on-line no valor de 100 dólares.

Em relação a confidencialidade, o estudo de Jiménez-López e Arenas Osuna (2017) demonstrou ser o mais frágil pois os dados dos residentes ficam armazenados na CSM-MR e o artigo não relata sobre a forma como os dados são armazenados, se são criptografados ou não. A CSM-MR é um espaço virtual, não possui uma infraestrutura física própria, utilizam o espaço no departamento das clínicas envolvidas no momento do estágio. Os responsáveis pela CSM-MR asseguram aos médicos residentes que as informações não são encaminhadas para o currículo acadêmico pessoal.

Quanto a participação dos médicos nos programas acima mencionados, o estudo realizado por Jiménez-López e Arenas Osuna (2017) obteve maior porcentagem de participantes, alcançou $98,6 \%$ e de acordo com os autores, as escalas de depressão, risco de suicídio e questionários sobre uso de substâncias anexadas nos papéis para matrícula favoreceram a obtenção das informações e identificação dos residentes em risco. $\mathrm{O}$ artigo foi o 
único que forneceu dados sobre o número de suicídios na instituição estudada antes da criação da CSM, possibilitando uma comparação dos resultados e eficácia do programa. A atuação da CSM-MR no período estudado apresentou o melhor resultado dentro do programa proposto. No entanto, do ponto de vista ético-médico-legal é questionável se esse estudo pode ser reproduzido em outras instituições pela ausência de elementos básicos para realização e publicação do artigo como o consentimento ou não do residente participar do programa.

Todos os programas foram elaborados após estudos por longos períodos sobre as possíveis causas do suicídio (Center et al., 2003). Cada instituição organizou um vasto programa com um grupo de especialistas e estudiosos para assegurar a confidencialidade, a privacidade e a eficácia. Apesar da preocupação em desenvolver um programa para identificação, prevenção e tratamento da saúde mental e suicídio, os artigos apresentaram um certo viés que pode ter contribuído no diagnóstico do estado da saúde mental e participação dos médicos. Inicialmente a análise ocorreu por meio do auto relato, sem avaliação de um especialista (psicólogo e/ou psiquiatra); a maior participação dos residentes no grupo da TCCi pode ter ocorrido pelo ganho de cartão-presente no valor de cem dólares; o melhor resultado do programa realizado por Jiménez-López e Arenas Osuna (2017) no México ocorreu por meio da identificação dos residentes mediante questionário sobre depressão, suicídio e uso de substâncias psicoativas e intervenção ativa da equipe da CSM-MR. Ver Quadro 2.

\section{Considerações finais}

A presente pesquisa sobre Prevenção do Suicídio em Médicos expôs uma história de longa data sobre o tema que cresceu de maneira alarmante no decorrer dos anos desde o primeiro caso publicado na América no século passado (Legha, 2012) até os dias atuais. Segundo os estudos realizados por especialistas preocupados com as elevadas taxas de mortalidade por suicídio, os transtornos mentais, sobretudo a depressão, foi e é considerada a maior responsável por essas mortes em médicos.

$\mathrm{Na}$ procura por artigos para prevenção e tratamento do suicídio em médicos, causou surpresa a escassez de publicações sobre o tema, apenas cinco artigos em 11 anos. De acordo com estudos anteriores, as principais causas foram reconhecidas há mais de 50 anos, no entanto os números de suicídio continuam elevados. A maioria dos artigos publicados sobre suicídio em médicos reconhece o transtorno mental principalmente a depressão como um fator de risco para o suicídio, mas o estigma, a vergonha, a falta de tempo, o receio de perder a licença para exercer a profissão ou o seguro profissional, a preocupação quanto ao registro do transtorno mental e/ou ideação suicida no seu prontuário médico têm impedido a procura por ajuda de um profissional. Diante desse problema, foram elaboradas estratégias para superar essas barreiras sem comprometer a confiabilidade, segurança, privacidade e a falta de tempo, que permitiriam ao médico buscar por ajuda/tratamento no completo anonimato se assim o desejasse. Os artigos mostraram pequena participação dos médicos (inferior a 15\%), mas com porcentagem elevada para depressão de moderado a alto e/ou suicídio. Apesar do pequeno número rastreado nos programas de saúde mental que buscaram por ajuda, os participantes se mostraram satisfeitos com o tratamento.

O esforço para criar um programa para prevenção do suicídio em médicos foi e é viável, mas ainda é necessário uma grande e abrangente mudança da comunidade médica quanto ao estigma do transtorno mental e suicídio.

Os artigos apresentaram estratégias isoladas para atender as necessidades das instituições, que podem ser considerados como ensaios bem conduzidos de programas direcionados para os médicos, mas não são consideradas estratégias universais de prevenção do suicídio.

Na pesquisa foi observada uma dificuldade dos médicos em geral de participar dos estudos para identificar e tratar possíveis transtornos mentais e ideação suicida, mas foi capaz de detectar médicos com alto risco para o suicídio e depressão grave dos participantes, entretanto poucos procuraram ou aceitaram tratamento. A principal barreira é o estigma e a relevância dessa causa é tão influente que até o presente momento, isto é, quase duas décadas depois do trabalho realizado por Center et al. (2003), as taxas de suicídio continuam elevadas e os estudos para diminuir o suicídio em médicos continuam.

A divulgação e compreensão do suicídio, reforçar que é prevenível e tratável desde que procure por ajuda, conscientização nas escolas médicas que estar em tratamento por transtorno mental e/ou por comportamento suicida (pensamento, tentativa) não o fará menos capacitado para exercer a medicina.

Esforços devem ser realizados na comunidade médica para combater o estigma da doença mental e do suicídio para vencer essa luta e desse modo diminuir a sua taxa.

A revisão permite realizar sugestões para um programa de prevenção de suicídio em médicos baseado nos artigos encontrados na pesquisa. Convidar para realizar 


\begin{tabular}{|c|c|c|c|c|c|}
\hline$\stackrel{\stackrel{\leftrightarrow}{>}}{>}$ & 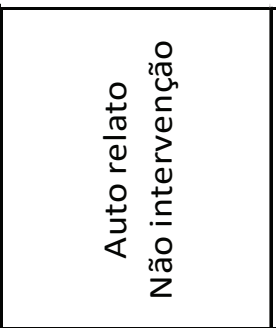 & 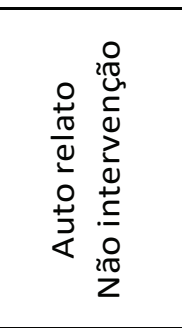 & 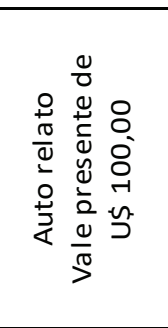 & 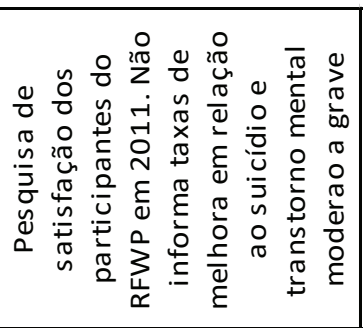 & 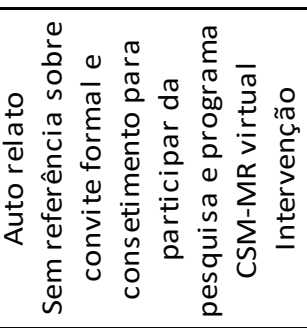 \\
\hline $\begin{array}{l}\frac{\pi}{u} \\
\frac{\Delta}{U} \\
\frac{u}{w}\end{array}$ & 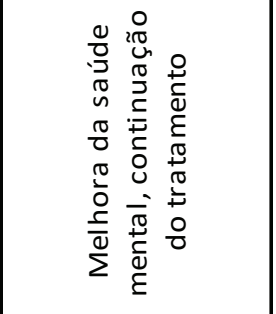 & 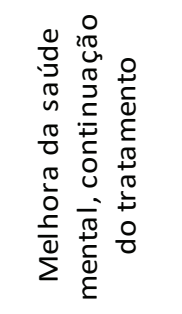 & 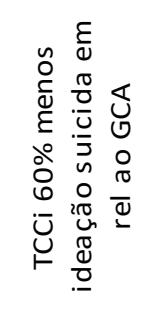 & 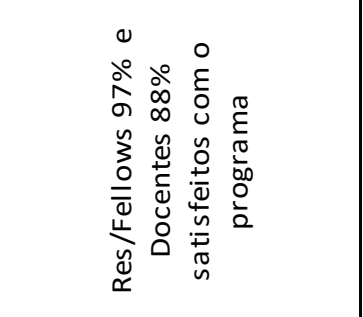 & 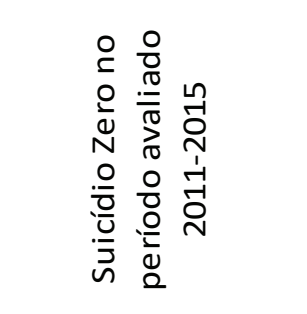 \\
\hline 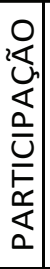 & 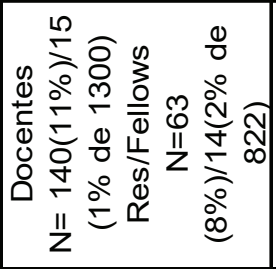 & 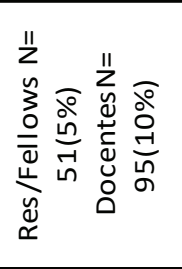 & 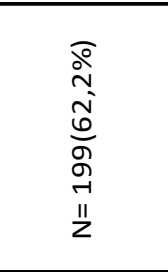 & 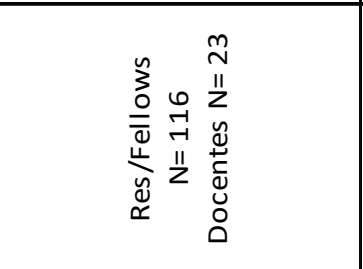 & 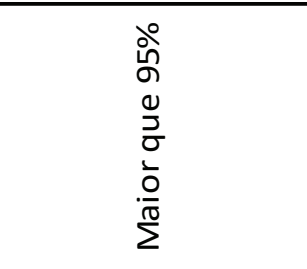 \\
\hline 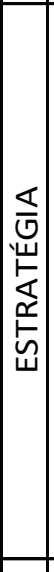 & 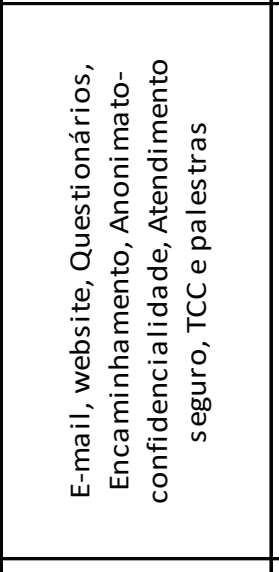 & 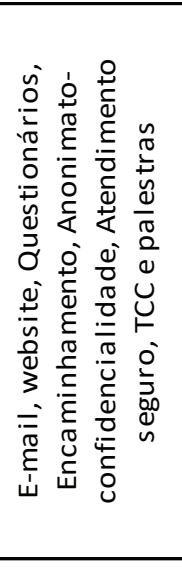 & 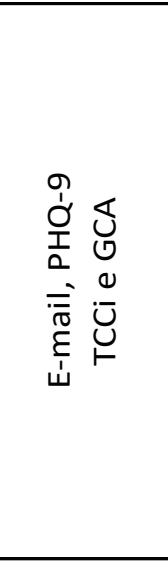 & 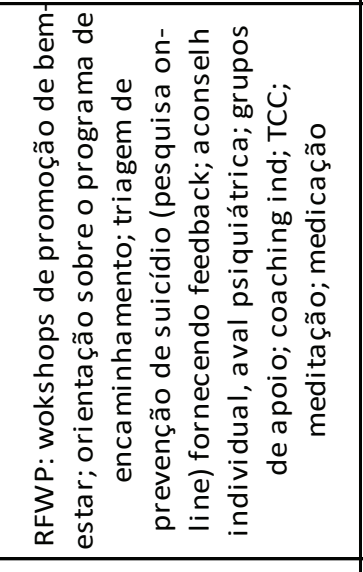 & 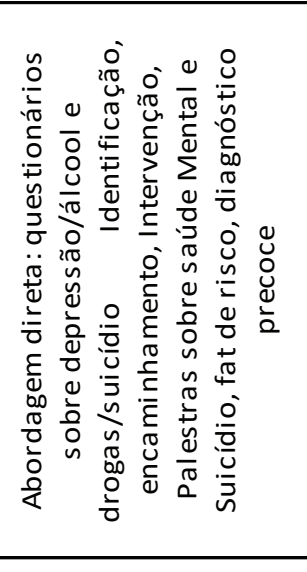 \\
\hline $\begin{array}{l}0 \\
2 \\
ن \\
4 \\
5 \\
5 \\
0 \\
0 \\
0\end{array}$ & 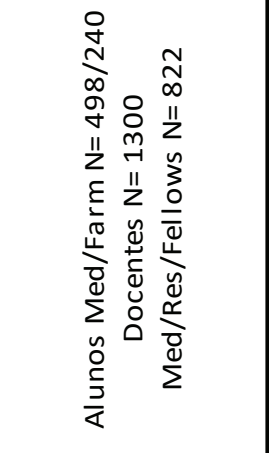 & 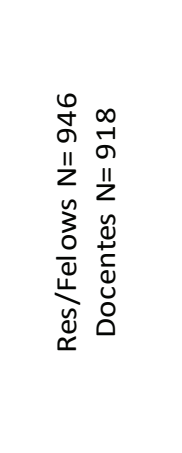 & 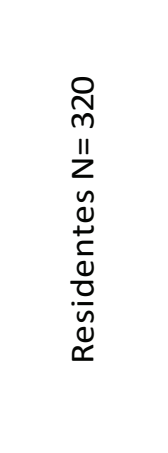 & 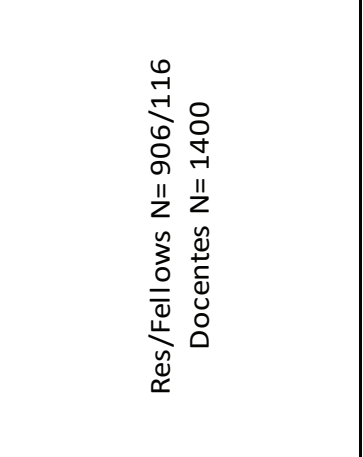 & 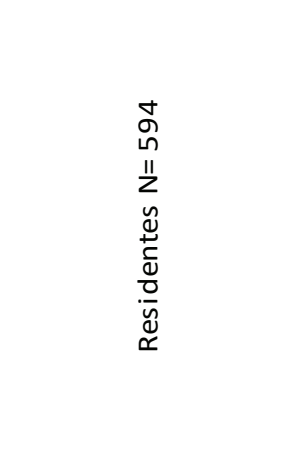 \\
\hline 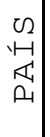 & گ্ & $\begin{array}{l}\mathbb{U} \\
\text { ป }\end{array}$ & 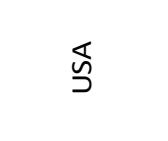 & $\begin{array}{l}\text { ऽ } \\
\text { }\end{array}$ & 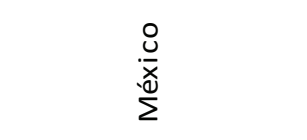 \\
\hline 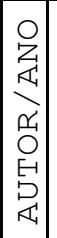 & 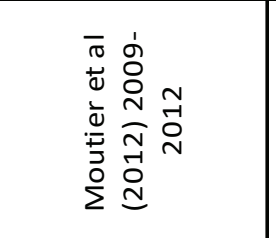 & 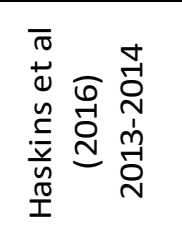 & 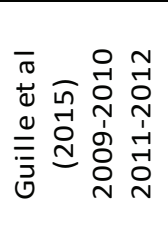 & 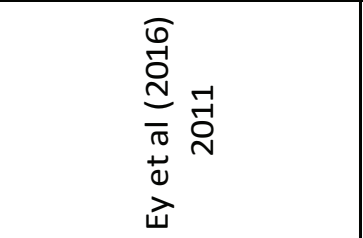 & 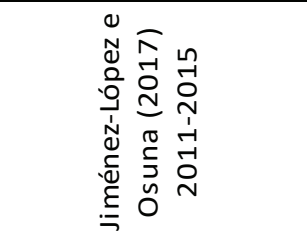 \\
\hline
\end{tabular}


uma pesquisa no momento da matrícula com os anexos (Jiménez-Lòpez, Arenas-Osuna \& Angeles-Garay, 2015) sobre depressão, ansiedade, ideação suicida, uso de substâncias psicoativas, antecedentes familiares de transtorno mental, se o residente está ou teve em algum transtorno mental, uso ou não de medicamento para distúrbios emocionais/mentais com garantia do anonimato, confiabilidade e que os dados não serão registrados nos arquivos da residência médica ou da instituição (Guille, et al., 2015; Haskins et al., 2016; Jiménez-Lòpez, Arenas-Osuna \& Angeles-Garay, 2015; Moutier et al., 2012) ou convidá-los por meio eletrônico para participar da pesquisa, obter o termo de consentimento e assegurar que os dados serão criptografados e não descodificável. Apresentar na aula inaugural uma palestra sobre uso e abuso de drogas / álcool, ideação suicida, estigma, depressão e outros transtornos mais comuns apresentados pelos médicos, quais os sinais e sintomas e como reconhece-los; indicar serviços de saúde mental das instituições próximo ao local de treinamento ou um mais distante (Ey, Moffit, Kinzie \& Brunett, 2016; Jiménez-Lòpez, Arenas-Osuna \& Angeles-Garay, 2015), oferecer palestras e workshops de promoção de bem-estar para residentes, fellows, corpo clínico e docentes (Ey, Moffit, Kinzie \& Brunett, 2016), apresentar a Terapia Cognitivo-Comportamental via internet (Guille et al., 2015), falar especificamente sobre depressão e desestigmatizar o transtorno mental e sua pior consequência, o suicídio (Moutier et al., 2012; Haskins et al., 2016; Ey, Moffit, Kinzie \& Brunett, 2016; Jiménez-Lòpez, Arenas-Osuna \& Angeles-Garay, 2015). Obter auxílio de um gatekeeper (observador) e dos chefes de disciplinas, docentes para identificar médico ou residente com transtorno mental e/ou risco de suicídio; apresentar lista de serviços de saúde mental e telefones pra contato (Guille et al., 2015), apresentar um website da instituição apresentando o programa de bem-estar, incluindo aí a prevenção do suicídio com questionários que abordarão transtornos mentais e suicídio; interação com o programa mantendo o anonimato se for o desejo do participante; em caso de risco de suicídio ou de uma depressão grave, convidá-lo para dialogar com o responsável pelo site anonimamente ou não, e/ou ser encaminhado para um especialista (Haskins et al., 2016; Jiménez-Lòpez, Arenas-Osuna \& Angeles-Garay, 2015; Moutier et al., 2012).

A leitura dos artigos revelou que esforços devem ser realizados para uma maior conscientização sobre suicídio e bem-estar (saúde mental), criação de programas em instituições de ensino médico e hospitais que ofereçam serviços variados para encaminhamento e tratamento no local ou em outro distante do trabalho, garantia de confidencialidade e segurança quanto aos dados obtidos para atingir o maior número de médicos para e diminuir as taxas de morte por suicídio entre eles.

\section{Referências}

AFSP (2015). Ten facts about physician suicide and mental bealth. https://afsp.org/ wp-content/uploads/2016/11/ten-facts-about-physician-suicide.pdf.

AFSP (2018). Facts about physician depression and suicide. American Foundation for Suicide Prevention. http://www.afsp.org/preventing-suicide/our-education-and-prevention-programs/programs-for-professionals/physician-and-medical-student-depression-and-suicide/facts-about-physician-depression-and-suicide.

Botega, N. J. (2015). Crise Suicida. 1 ed. Porto Alegre: Artmed.

Botega N. J., Werlang B. S. G. \& Macedo M. M. K. (2006). Prevenção do comportamento suicida. Psico. Porto Alegre; v. 37, n. 3, p. 213-220. http://revistaseletronicas.pucrs.br/ojs/index.php/revistapsico/article/viewFile/1442/1130.

BRASIL (2009). Ministério da Saúde. Secretaria de Vigilância em Saúde. Manual instrutivo de preenchimento da ficha de notificação/investigação de violência doméstica, sexual e outras violências. Brasília. http://www.saude.gov.br/images/pdf/2016/ fevereiro/16/instrutivo-ficha-sinan-5-1--vers--o-final-15-01-2016.pdf.

Cassorla, R. (1984). O que é suicídio? (p.9)1ª ed. São Paulo: Editora Brasiliense. https://issuu.com/rafaelcrisostomo1/docs/o_que____suic__dio_-_ roosevelt_cass.

Center, C., Davis, M., Detre, T., Ford, D. E., Hansbrough, W., Hendin, H., Laszlo, J., Litts, D. A., Mann, J., Mansky, P. A., Michels, R., Miles, S. H., Proujansky, R., Reynolds, C. F., 3rd \& Silverman, M. M. (2003). Confronting depression and suicide in physicians: a consensus statement. JAMA, 289(23), 3161-3166. https://doi.org/10.1001/jama.289.23.3161.

Chachamovich, E., Stefanello, S., Botega N. \& Turecki, G. (2009). Quais são os recentes achados clínicos sobre a associação entre depressão e suicídio? Rev Bras Psiquiatr.31(Supl I):S18-25.

Ey, S., Moffit, M., Kinzie, J. M. \& Brunett, P. H. (2016). Feasibility of a comprehensive wellness and suicide prevention program: a decade of caring for physicians in training and practice. Journal of graduate medical education, 8(5), 747-753. https://pubmed.ncbi.nlm.nih.gov/28018541/.

Gerada, C. (2018b). Doctors, suicide and mental illness. B.J.Psych Bulletin, 42(4), 165-168. doi:10.1192/bjb.2018.11.

Gold, K. J., Sen, A. \& Schwenk, T. L. (2013). Details on suicide among U.S. physicians: data from the National Violent Death Reporting System. Gen Hosp Psychiatry. 35(1):45-9.

Guille, C., Zhao, Z., Krystal, J., Nichols, B., Brady, K. \& Sen, S. (2015). Web-Based Cognitive Behavioral Therapy Intervention for the Prevention of Suicidal Ideation in Medical Interns: A Randomized Clinical Trial. JAMA psychiatry, 72(12), 1192-1198. https://doi.org/10.1001/jamapsychiatry.2015.1880.

Gunter, T. (2016). Physician Death by Suicide: Problems Seeking Stakeholder Solutions. Indiana University Robert H. McKinney School of Law Research Paper $N^{o}$ 2016-23. https://ssrn.com/abstract=2761854 or http://dx.doi. org/10.2139/ssrn.2761854.

Haskins, J., Carson, J. G., Chang, C. H., Kirshnit, C., Link, D. P., Navarra, L., Scher, L. M., Sciolla, A. F., Uppington, J. \& Yellowlees, P. (2016). The Suicide Prevention, Depression Awareness, and Clinical Engagement Program for Faculty and Residents at the University of California, Davis Health System. Academic psychiatry: the journal of the American Association of Directors of Psychiatric Residency Training and the Association for Academic Psychiatry, 40(1), 23-29. https://doi.org/10.1007/s40596-015-0359-0.

Hawton, K., Malmberg, A. \& Simkin, S. (2004). Suicide in doctors. Journal of Psychosomatic Research, v. 57, n. 1, p. 1-4. https://doi:10.1016/s00223999(03)00372-6.

Jiménez-Lòpez, J. L., Arenas-Osuna, J. y Angeles-Garay, U. (2015). Sintomas de depresión, ansiedad y riesgo de suicidio en médicos residentes durante un año académico. Revista Médica del Instituto Mexicano del Seguro Social, 53(1), 20-28. http://www.medigraphic.com/pdfs/imss/im-2015/im151d.pdf.

Jiménez-López, J. L. \& Arenas Osuna, J. (2017). Programa de prevención del suicidio, tratamiento de trastornos mentales y promoción de la salud mental para médicos 
residentes / Program for suicidal prevention, mentaldisordertreatment, and mentalhealth development for residente doctors. Revista Colombiana de Ciencias Sociales, 8(1), pp. 227-241. DOI: http://dx.doi.org/10.21501/22161201.1819.

Legha, R. K. A (2012). History of Physician Suicide in America. J. Med Humanit, v. 33, n. 4, p. 219-244. https://doi 10.1007/s10912-012-9182-8.

Meleiro, A. M. S. (1998). Suicídio entre médicos e estudantes de medicina. Rev Assoc Med Bras, São Paulo, v. 44, n. 2, p. 135-140. http://www.scielo. br/scielo. php?script=sci_arttext\&pid=S0104-42301998000200012\&ln $\mathrm{g}=\mathrm{pt} \& n \mathrm{~mm}=$ iso\&tlng $=\mathrm{pt}$.

Millan, L. R. \& Arruda, P. C. V. (2008). Assistência psicológica ao estudante de Medicina: 21 anos de experiência. Revista da Associação Médica Brasileira, São Paulo, v. 54, n. 1, p. 90-94. http://dx.doi.org/10.1590/S0104-42302008000100027.

MS (Ministério da Saúde). (2014). http://www.saude.gov.br/images/pdf/2017/ setembro/21/2017-025-Perfil-epidemiologico-das-tentativas-e-obitos-por-suicidio-no-Brasil-e-a-rede-de-atencao-a-saude.pdf.

Moutier, C., Norcross, W., Jong, P., Norman, M., Kirby, B., McGuire, T. \& Zisook, S. (2012). The suicide prevention and depression awareness program at the University of California, San Diego School of Medicine. Academic Medicine, 87(3), 320-326. https://journals.lww.com/academicmedicine/ FullText/2012/03010/The_Suicide_Prevention_and_Depression_Awareness.18.aspx?casa_token $=V W \lg X \mathrm{mAhNDEAAAAA:PTBJt-YMZseEAk-}$ n7eDXgxW7E0bTrEkN4g7Y0-yvu2qYxgi9kSUBYq8hF7py0h8aYrDix6wiEVmkHOcNqbYPRpPsziYliTfIN.
OMS (Organização Mundial da Saúde). (2014). Preventive suicide: a global imperative. Geneva: OMS. https://www.who.int/mental_health/suicide-prevention/ world_report_2014/en/.

OMS (Organização Mundial da Saúde). (2000). Prevenção do suicídio: um manual para médicos clínicos gerais [online]. Genebra: OMS. https:/www.who.int/ mental_health/prevention/suicide/en/suicideprev_gp_port.pdf.

Santa, N. D. \& Cantilino, A. (2016). Suicídio entre Médicos e Estudantes de Medicina: Revisão de Literatura. Rev. Bras. Educ. Med.; Rio de Janeiro, v. 40,n. 4, p. 772-780. http://dx.doi.org/10.1590/1981-52712015v40n4e00262015.

Scavacini K. (2018). O suicídio é um problema de todos: a consciência, a competência e o diálogo na prevenção e posvenção. Tese de doutorado.

Sen, S, Kranzler, H. R., Krystal, J. H., Speller, H. Chan, G. et al. (2010). A prospective cohort study investigating factors associated with depression during medical internship. Arch Gen Psychiatry. 67(6):557-565. https://pubmed.ncbi.nlm. nih.gov/20368500/.

Sheikhmoonesi, F. \& Zarghami, M. (2014). Prevention of physicians' suicide. Iran. J. psychiatry Behav. Sci. 8 (2): 1-3. https://www.ncbi.nlm.nih.gov/pmc/ articles/PMC4105598/.

Valladolid, M. N. (2011). Suicidio. Revista Peruana de Epidemiología, 15(2), 1-5. https://dialnet.unirioja.es/servlet/articulo?codigo $=3994798$.

Submetido em: 22-9-2020

Aceito em: 9-4-2021 